\title{
Detection of Swine Dysentery (SD) caused by Brachyspira hyodysenteriae via PCR in Northern Vietnam
}

\section{Nguyen Thi Thu Hang, Truong Quang Lam, Dao Le Anh, Nguyen Thi Hoa, Nguyen Thi Lan \& Nguyen Thi Thuy Dung}

Faculty of Veterinary Medicine, Vietnam National University of Agriculture, Hanoi 131000, Vietnam

\begin{abstract}
This study aimed to diagnose swine dysentery (SD) caused by Brachyspira hyodysenteriae in pigs by the PCR method in Vietnam. Of the 250 samples, 29 isolates of $B$. hyodysenteriae (11.60\%) were identified by PCR in seven provinces of Northern Vietnam, and the infection rate differed from region to region. From the positive cases of $B$. hyodysenteriae, we analyzed $B$. hyodysenteriae infected cases according to the ages of the pigs, farm sizes, and veterinary hygiene practices to get more information about the disease in Vietnam. The results showed that the positive $B$. hyodysenteriae samples were commonly seen in post-weaning pigs $(32.14 \%)$ in households (20.73\%) with poor hygiene (24.69\%). Clinical signs of SD included high fever (100\%); anorexia (100\%); watery, bloody diarrhea, usually gray to brown in color (100\%); and weight loss $(86.42 \%)$. Gross lesions of SD were limited to the large intestine were described as having a fibrinous, blood-flecked membrane covering the mucosa $(93.75 \%)$, swollen with hemorrhaged colon and cecum $(75.00 \%)$, and mesenteric lymph nodes $(81.25 \%)$
\end{abstract}

\section{Keywords}

Swine dysentery, Brachyspira hyodysenteriae, PCR, Vietnam

\section{Introduction}

Swine dysentery (SD) was first recorded in the mid-west of the

Received: October 27, 2020 Accepted: June 6, 2021

\section{Correspondence to}

Dao Le Anh

daoleanh@vnua.edu.vn

\section{ORCID}

Dao Le Anh https://orcid.org/0000-0002-05308309 USA in 1921 (Whiting et al., 1921), however the etiology remains unknown. After 50 years, Brachyspira hyodysenteriae was confirmed as the causative agent of swine dysentery (Taylor \& Alexander, 1971). $B$. hyodysenteriae is a gram-negative, motile, helically coiled (spiralshaped), anaerobic bacterium. It is $6.0-8.5 \mu \mathrm{m}$ long, $0.32-0.38 \mu \mathrm{m}$ wide, and has 7-14 periplasmic flagella inserted at each cell end. The cell is covered by a loose outer membrane. The outer envelope of $B$. hyodysenteriae contains lipooligosaccharides (LOS), a semi-rough form of lipopolysaccharides (Hampson et al., 2006). 
main clinical sign of SD is diarrhea, and gray to yellow mucoid feces is usually the first sign noticed. With SD, diarrhea continues and quickly becomes mucohemorrhagic, with excess mucus and fresh blood apparent. It is one of the most common intestinal infections in pigs worldwide. The prevalence of the infection varies from country to country, for example, SD accounts for $2.5 \%$ of all pig infections in Europe, $14.0 \%$ in Denmark, $13 \%$ in the United Kingdom, $10.81 \%$ in Italy, and 32.10\% in Spain (Møller et al., 1998; Stege et al., 2000; Thomson et al., 2001; Merialdi et al., 2003; Carvajal et al., 2006). Variations in prevalence can be due to the use of different diagnostic methods or to differences among countries in housing, management, feeding regimes, etc. (Jacobson et al., 2005). SD has also been reported in South America and Southeast Asia (Burrough, 2016). Treating SD involves the use of antibiotics. Pleuromutilins (tiamulin and valnemulin) have been used for this purpose in the European Union (EU). Also, macrolides (tylosin and, more recently, tylvalosin) and the closely related lincomycin (lincosamide) have been commonly included in SD therapeutic strategies (Hampson et al., 2006).

According to Vu Dinh Hung and Doan Khac Huc (1996), SD was determined in some industrial rearing farms in Vietnam many years ago, but there is no official information of SD in pigs in Vietnam at present. There are also not any published articles discussing the existence and prevalence of SD in Vietnam now.

The advent of molecular diagnostic methods, such as polymerase chain reaction (PCR) targeting known pathogenic Brachyspira spp., are being applied in many laboratories around the world because of the advantages of high sensitivity, specificity, and fast diagnosis.

In the Red River Delta, there are livestock farms in general and pig farms in particular occupying a large proportion of the region that are becoming increasingly more important in terms of their position in Vietnamese agriculture. However, due to many limitations on small-scale breeding, utilization of and lack of technology, and environmental sanitation, there is a potential risk of disease outbreak. We aimed to determine the existence and prevalence of $B$. hyodysenteriae from fecal samples of pigs in some northern provinces of Vietnam by the PCR technique. Our findings would provide valuable information about the effective prevention and treatment options of $B$. hyodysenteriae for farmers, veterinarians, and universities.

\section{Materials and Methods}

\section{Collecting samples}

From August 2018 to August 2020, 250 samples were collected from 40 farms of 20 villages in seven provinces of Northern Vietnam (Hai Duong, Thai Binh, Hung Yen, Ha Nam, Bac Giang, and Bac Ninh). The feces and rectal swab samples were collected from pigs that showed symptoms of fever, moodiness, anorexia, and diarrhea. Samples were taken from pigs of all growth stages (65 newborn pigs, 56 postweaning pigs, 66 growing pigs, and 63 sows) and from various types of farming systems (household, semi-intensive, and intensive). The samples were transported directly to the Key Laboratory of Veterinary Biotechnology, Vietnam National University of Agriculture for examination.

Questionnaires and direct interviews with farmers were used to gain knowledge about clinical signs, breeding methods, and veterinary hygiene, etc. The selection of $16 \quad B$. hyodysenteriae positive cases with typical clinical signs were slaughtered to analyze their gross pathology.

\section{PCR analysis}

DNA from the feces and rectal swabs was extracted using a Dneasy Blood \& Tissue Kit (Qiagen - 69506.250). For amplification of the conserved genes (nox) of B. hyodysenteriae, the PCR primers reported by Tom La et al. (2003) were used with the sequences BF: ACTAAAGATCCTGATGTATTG and BR: CTAATAAACGTCTGCTGC. The $25 \mu \mathrm{L}-$ reaction mixture consisted of $10 \mu \mathrm{M}$ of each primer $\mathrm{BF} / \mathrm{BR}, 2.5 \mathrm{mM} \mathrm{MgCl}, 200 \mu \mathrm{M}$ each of dNTP, 1UI of Taq DNA polymerase (GoTaq Green_Promega Kit), 1X PCR buffer, and $5 \mu \mathrm{L}$ of the extracted DNA. Amplification was carried out in a thermal cycler with an initial 
denaturation at $94^{\circ} \mathrm{C}$ for $5 \mathrm{~min}$, followed by 35 cycles of denaturation at $94^{\circ} \mathrm{C}$ for $60 \mathrm{~s}$, annealing at $52^{\circ} \mathrm{C}$ for $60 \mathrm{~s}$, and extension at $72^{\circ} \mathrm{C}$ for $90 \mathrm{~s}$, and a final extension step at $72^{\circ} \mathrm{C}$ for $7 \mathrm{~min}$.

\section{Data analysis}

The data were analysed using Microsoft Office Excel 2010 software with the Chi-square statistic.

\section{Results}

\section{Effect of doses and ratios of mineral fertilizer on growth of PH10 tea variety}

Of the 250 samples, 29 positive cases of $B$. hyodysenteriae $(11.60 \%)$ were identified by PCR (Table 1). B. hyodysenteriae was seen in all seven of the tested provinces (Hai Duong, Thai Binh, Hung Yen, Ha Nam, Bac Giang, Thai Nguyen, and Bac Ninh). The highest infection rate of $B$. hyodysenteriae was $17.14 \%$ in Hai Duong province, followed by Thai Binh, Hung Yen, Bac Giang, and Bac Ninh provinces (13.0415.79\%), while $\mathrm{Ha} \mathrm{Nam}$ and Thai Nguyen provinces were infected with $B$. hyodysenteriae at the lowest rates $(2.78 \%$ and $3.57 \%$, respectively). These differences could be due to different farming conditions, feed, techniques, and epidemiology in each local. The physical geographic conditions of each local also contributed to the differences, and depending on the time of the investigation, weather factors could have altered the resistance of the animals.

Table 1. The positive cases of $B$. hyodysenteriae by PCR

\begin{tabular}{|c|c|c|c|}
\hline \multirow{2}{*}{ Region } & \multirow{2}{*}{ Total } & \multicolumn{2}{|c|}{ PCR } \\
\hline & & Positive & $\%$ \\
\hline Hai Duong & 35 & 6 & 17.14 \\
\hline Thai Binh & 37 & 5 & 13.51 \\
\hline Hung Yen & 38 & 6 & 15.79 \\
\hline $\mathrm{Ha} \mathrm{Nam}$ & 36 & 1 & 2.78 \\
\hline Bac Giang & 46 & 6 & 13.04 \\
\hline Bac Ninh & 30 & 4 & 13.33 \\
\hline Thai Nguyen & 28 & 1 & 3.57 \\
\hline Total & 250 & 29 & 11.60 \\
\hline
\end{tabular}

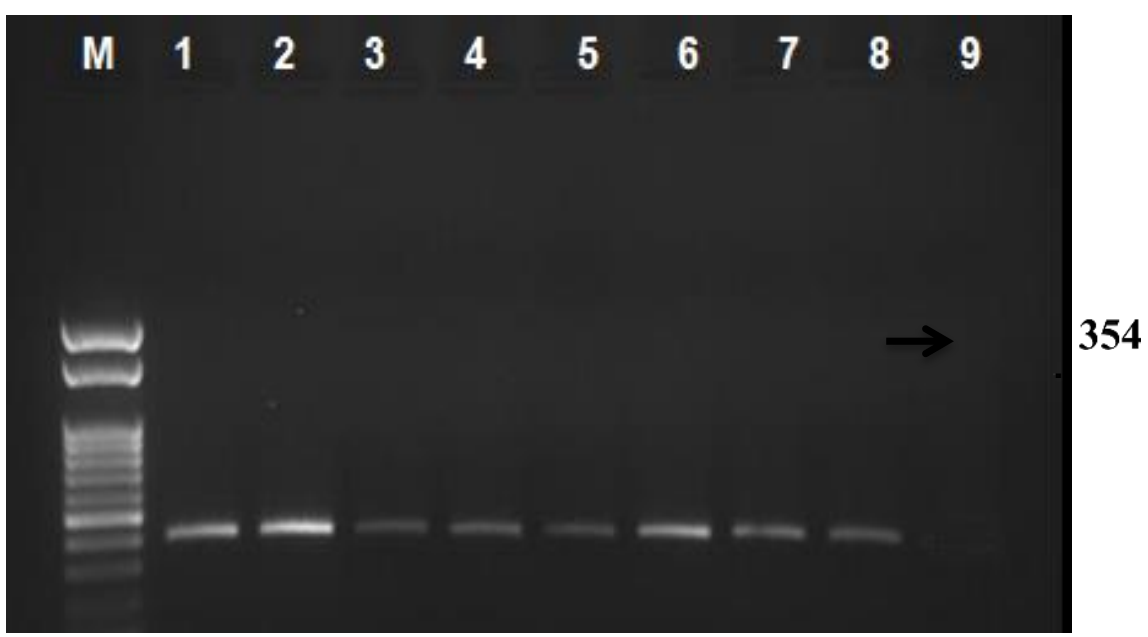

Figure 1. Running gel of PCR products

Lane M: Marker; Lanes 2 to 8: The positive samples of B. hyodysenteriae (No. 1, 2, 3, 4, 5, 6, 7); Lane 1: Positive control; Lane 9: Negative control 
These findings show that $B$. hyodysenteriae is common in pigs in northern provinces of Vietnam.

Once the positive cases of $B$. hyodysenteriae were identified, these cases were analyzed according to the age of the pigs and farm size (Table 2), veterinary hygiene (Table 3), and season (Table 4) to get more information about SD in Vietnam.

B. hyodysenteriae infected pigs in all growth stages (newborn pigs, post-weaning pigs, grower pigs, and sows). In particular, post-weaning pigs suffered from SD the most with an infection rate of $32.14 \%$, followed by grower pigs $(10.61 \%)$. The lowest infection rate belonged to newborn pigs and sows (3.08 and 3.17\%, respectively) (Table 2).

B. hyodysenteriae was seen in all three types of farming systems investigated. As such, households had the highest infection rate of $\mathrm{B}$. hyodysenteriae (20.73\%), followed by the semi- intensive scale (12.05\%). The lowest infection rate was seen in intensive farming $(2.35 \%)$.

Farms that had a lower frequency of veterinary hygiene, had the highest infection rate of B. hyodysenteriae (24.69\%). As the frequency of veterinary hygiene increased, the infection of SD decreased (2.35-8.33\%). In this study, we assessed that the risk of SD infection was reduced in farms with good veterinary hygiene.

\section{Clinical signs of swine dysentery (SD)}

Twenty-nine positive samples of swine dysentery were identified by PCR and RT-PCR to detect co-infection with other diarrhea agents (Lawsonia, Salmonella, E.coli, Clostridium perfingens, PEDV- Porcine Epidemic Diarrhoea virus, and Isospora suis). The results showed that 26 pigs were only infected with SD (data not shown). Some of the clinical signs of the 26 infected pigs are listed in Table 4.

Table 2. The positive cases of $B$. hyodysenteriae by age group and farm size

\begin{tabular}{|c|c|c|c|c|c|c|c|c|c|c|c|c|}
\hline \multirow{3}{*}{ Growth stage } & \multirow{3}{*}{$\mathrm{N}$} & \multirow{3}{*}{$\mathrm{P}$} & \multirow{3}{*}{$\%$} & \multicolumn{9}{|c|}{ Farm size } \\
\hline & & & & \multicolumn{3}{|c|}{ Household } & \multicolumn{3}{|c|}{ Semi-intensive } & \multicolumn{3}{|c|}{ Intensive } \\
\hline & & & & $\mathrm{N}$ & $\mathrm{P}$ & $\%$ & $\mathrm{~N}$ & $\mathrm{P}$ & $\%$ & $\mathrm{~N}$ & $\mathrm{P}$ & $\%$ \\
\hline Newborn & 65 & 2 & 3.08 & 20 & 1 & 5.00 & 22 & 1 & 4.55 & 23 & 0 & 0 \\
\hline Post-weaning & 56 & 18 & 32.14 & 22 & 10 & 45.45 & 16 & 7 & 43.75 & 18 & 1 & 5.56 \\
\hline Grower & 66 & 7 & 10.61 & 21 & 5 & 23.81 & 25 & 1 & 4.00 & 20 & 1 & 5.00 \\
\hline Sows & 63 & 2 & 3.17 & 19 & 1 & 5.26 & 20 & 1 & 5.00 & 24 & 0 & 0 \\
\hline Total & 250 & 29 & 11.60 & 82 & 17 & $20.73^{a}$ & 83 & 10 & $12.05^{\mathrm{b}}$ & 85 & 2 & $2.35^{\mathrm{c}}$ \\
\hline
\end{tabular}

Note: Household: <30 pigs/farm; Semi-intensive: $31-300$ pigs/farm; Intensive: >300 pigs/farm

Superscripts $a, b$, and $c$ indicate statistically significant differences $(P<0.05)$

N: Investigated number; P: Positive number; \%: Percentage

Table 3. The positive cases of $B$. hyodysenteriae by age group and veterinary hygiene

\begin{tabular}{|c|c|c|c|c|c|c|c|c|c|}
\hline \multirow{3}{*}{ Growth stage } & \multicolumn{9}{|c|}{ Hygiene } \\
\hline & \multicolumn{3}{|c|}{ Rarely } & \multicolumn{3}{|c|}{ Sometimes } & \multicolumn{3}{|c|}{ Usually } \\
\hline & $\mathrm{N}$ & $\mathrm{P}$ & $\%$ & $\mathrm{~N}$ & $\mathrm{P}$ & $\%$ & $\mathrm{~N}$ & $\mathrm{P}$ & $\%$ \\
\hline Newborn & 21 & 2 & 9.52 & 19 & 0 & 0 & 23 & 0 & 0 \\
\hline Post-weaning & 19 & 14 & 73.68 & 18 & 3 & 16.67 & 18 & 1 & 5.56 \\
\hline Grower & 22 & 2 & 9.09 & 22 & 4 & 18.18 & 20 & 1 & 5.00 \\
\hline Sows & 19 & 2 & 10.53 & 25 & 0 & 0 & 24 & 0 & 0 \\
\hline Total & 81 & 20 & $24.69^{a}$ & 84 & 7 & $8.33^{b}$ & 85 & 2 & $2.35^{c}$ \\
\hline
\end{tabular}

Note: N: Investigated number; P: Positive number; \%: Percentage

Superscripts $a, b, c$ indicate statistically significant differences $(P<0.05)$. 
Table 4. Prevalence of clinical signs of swine dysentery

\begin{tabular}{lcc}
\hline \multicolumn{1}{c}{ Clinical signs } & No $(\mathrm{n}=26)$ & $\%$ \\
\hline High fever $\left(40.0-41.5^{\circ} \mathrm{C}\right)$ & 26 & 100 \\
Anorexia, moodiness & 26 & 100 \\
Mucoid diarrhea with grey - brown or bloody feces & 26 & 100 \\
Dry skin, weight loss & 19 & 73.08 \\
\hline
\end{tabular}
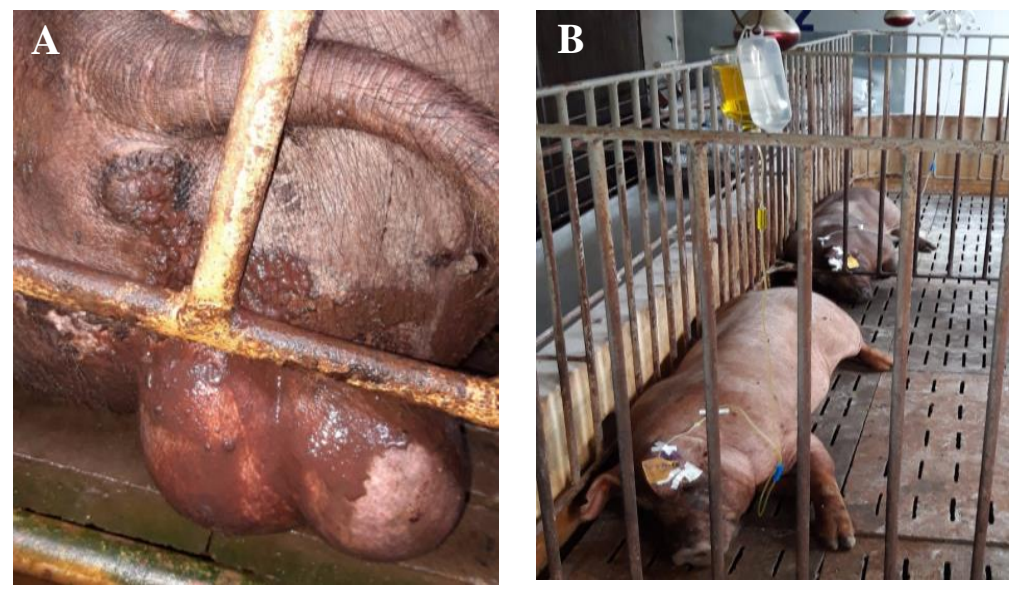

Figure 2. Clinical signs of Swine dysentery (A. Diarrhea pigs, bloody feces; B. Tired pigs)

All the pigs infected with $B$. hyodysenteriae had a high fever and mucoid diarrhea with grey brown or bloody feces (Figure 2). Other clinical symptoms were described including the pigs being emaciated, having dry skin, and significant weight loss.

A high fever of $40.0-41.5^{\circ} \mathrm{C}$ was reported as the first sign of swine dysentery. Although a fever is not an exclusive symptom for a particular disease, it signaled that the pigs were responding to the causative agent. Meanwhile, other symptoms such as moodiness and fatigue were typically seen alongside the fever. These findings are in complete agreement with a previous study of SD caused by B. hyodysenteriae in India (Walczak, 2015).

SD caused diarrhea with variations in color (grey-brown or bloody) and often contained mucus or blood. Watery diarrhea or loose feces that was brown to gray were observed more frequently in piglets 7 to 14 days old. As the disease progressed, the intestinal epithelial cells were destroyed, which then produced mucus and changes in blood flow causing bloody feces (Hampson, 2012).
Body mass reductions and dry skin occurred when the pigs experienced prolonged diarrhea, which led to the pigs falling into dehydration and severe anemia situations. The gastrointestinal tract of the pigs was damaged by inflammation and necrosis. It affected the intestinal function of the body's absorption; therefore, nutrient absorption was significantly reduced making the body skinny. In addition, the large intestine was the most affected organ as water reabsorption was delayed here leading to electrolyte disturbances and dehydration in animals with severe diarrhea.

\section{Gross pathology of Swine dysentery}

The gross lesions of SD have been commonly described as including swelling in the large intestine (56.25\%), especially in the colon and cecum. Besides, severe hemorrhaging and swelling in the colon and cecum have also been mentioned as aspects of typical gross lesions of SD (Table 5). As the large intestine was affected, the inside lining of the intestine became destroyed and necrotic. As a result, the intestinal mucosa was hemorrhaged and covered with mucus (93.75\%) (Figure 3). However, the mechanisms of tissue 
Table 5. Gross lesions of swine dysentery

\begin{tabular}{lcc}
\hline \multicolumn{1}{c}{ Gross lesions } & No. $(\mathrm{n}=16)$ & $\%$ \\
\hline Swelling in large intestine & 9 & 56.25 \\
Swelling and hemorrhaging in colon and cecum & 12 & 75.00 \\
Swollen mesenteric lymph nodes & 13 & 81.25 \\
Fibrinous, blood-flecked membrane covering the mucosa & 15 & 93.75 \\
\hline
\end{tabular}
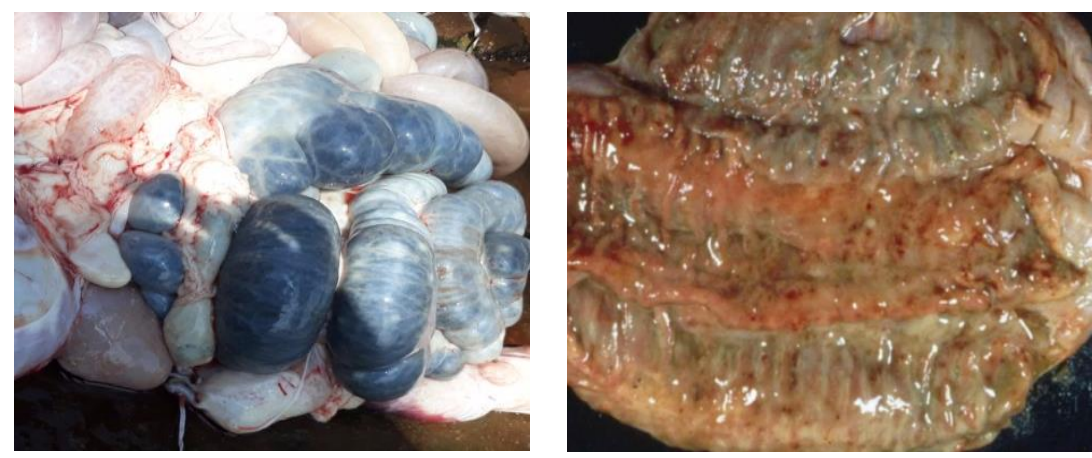

Figure 3. Gross lesions of swine dysentery

A. Swelling of the large intestine; B. The mucosa of the colon is covered with mucus and blood

destruction in $B$. hyodysenteriae have not been fully elucidated. Hemolysin(s) and LOS may play a role by acting locally to disrupt the epithelial barrier in the colon, resulting in epithelial sloughing (Hampson, 2012).

When pigs are infected with $B$. hyodysenteriae, water reabsorption in the large intestine is also affected. Therefore, while the animal suffered from severe diarrhea, fluid and mucus were found in the intestines. In severe cases of infection, intestinal mucosa covered with fibrin mixed with blood were observed. The gross lesions of swine dysentery in this study coincide with the findings of other scientists from around the world (Burrough, 2016; Szancer, 2019).

\section{Discussion}

SD caused by $B$. hyodysenteriae has been reported worldwide, however, reliable prevalence figures are limited. There is less information about SD in Asian countries, but recently, B. hyodysenteriae was detected in Hong Kong and Taiwan (Yeh et al., 2018; Lugsomya et al., 2019). This is the first study to accurately detect the prevalence of $B$. hyodysenteriae in the North of Vietnam by the PCR technique.
Twenty-nine cases were determined positive for B. hyodysenteriae from the 250 pigs sampled in seven northern provinces, accounting for $11.60 \%$ and showing the relatively common circulation of B. hyodysenteriae in Northern Vietnam. The rate of SD infection in Asia has not been the topic of many publications. Data on the prevalence of SD in Vietnam are similar to studies on prevalence rates in European countries (Thomson et al., 2001; Merialdi et al., 2003; Carvajal et al., 2006). Analysis of the $B$. hyodysenteriae positive cases showed that $\mathrm{SD}$ is distributed in pigs of different ages, across different seasons, and in households/farms with poor hygiene practices.

These results showed that the prevalence of SD differed between growth stages, and postweaning pigs have a higher risk of infection SD $(32.14 \%)$ than other ages $(3.08-10.61 \%)$. There is no $B$. hyodysenteriae vaccine yet, so in farms where pathogens have been circulating, $B$. hyodysenteriae antibodies can exist in sows (carriers) which can then be passed from sows to newborns pigs. In post-weaning piglets, the level of antibodies transmitted by sows is reduced to the lowest level, so the risk of SD infection at this age period is larger than in other ages. Moreover, the immune responses of pigs at the post- 
weaning stage are not high. If pigs are not provided with proper nutrition to increase resistance or are raised in an unsanitary environment, they are more easily infected with bacteria (Ochiai et al., 1997). In naive weaned pigs, the morbidity and mortality of SD can approach 90\% and 30\%, respectively (Hampson, 2012).

In this study, the number of SD-positive cases due to B. hyodysenteriae was highest in households (58.62\%), corresponding with those who raise pigs with poor veterinary hygiene $(68.97 \%)$. It is true that the household is the most common type of farming practice in Vietnam and the infection rate is affected by traditional breeding methods, poor biosecurity, and poor hygiene due to pigs within the same age group being raised in the same room without being categorized for each growth stage. The transmission vectors of the disease are mice and birds, and they are considered the major causes of disease spread. The majority of diseases are considered to be derived from household farms, especially infectious diseases in Vietnam.

$B$. hyodysenteriae is able to survive in the environment of the farm for a long period of time, and B. hyodysenteriae is relatively resistant in the environment of a pig house. It can survive in soil held at $10^{\circ} \mathrm{C}$ for 10 days. In the presence of feces, the survival time is increased to 78 days, and even can reach 112 days in pure pig feces (Boye, 2011). Also, B. hyodysenteriae was able to survive for 48 days in dysenteric feces held between 0 and $10^{\circ} \mathrm{C}$, although it only survived for 7 days at $25^{\circ} \mathrm{C}$, and for less than $24 \mathrm{~h}$ at $37^{\circ} \mathrm{C}$ (Chia \& Taylor, 1978). Poor hygiene, overstocking, transportation, and cold temperatures have been described as the predisposing factors to the development of SD in endemically infected farms. Rigid hygiene plays an important role in the prevention and control of bacterial diseases. The environment should be cleaned regularly not only to limit transmission but also to destroy the pathogens hiding around the farming area. Bacteria are easily destroyed by common disinfectants or under sunlight (Harris, 1984).

Total depopulation, cleanup, disinfection, and repopulation with an SD-free stock is sometimes the only method available to eliminate $B$. hyodysenteriae; however, it should only be instituted after accurate financial calculations.

Moreover, rodents are potential vectors of Brachyspira spp. Some insect species can carry important enteric disease agents with implications for on-farm spread and maintenance, affecting biosecurity and eradication protocols on pig farms. Insect vectors seem to harbor Brachyspira spp. and act as a reservoir and source of infection for pigs. $B$. hyodysenteriae has been isolated from cockroaches and flies. The climate of Vietnam is quite favorable for rodents and insects to grow. Therefore, hygiene to destroy pathogens and vectors is very important and directly affects the transmission of disease between individuals in a passel. Thus, pig farmers need to strictly implement veterinary and barn hygiene, and kill intermediate vectors such as mice, flies, etc. all year to prevent SD disease caused by $B$. hyodysenteriae.

From the analysis of the clinical signs, the gross lesions of the $B$. hyodysenteriae positive cases show that it is difficult to distinguish the disease from many other causes of diarrhea in pigs if only by observation and necropsy. Academically, a number of enteric diseases might be confused with SD which often coincides with other enteric infections (Møller et al., 1998; Thomson et al.,1998). Some diseases caused by Lawsonia intracellularis, Salmonella, trichuriasis, or gastric ulcers have clinical symptoms and lesions in the large intestine similar to SD (Hampson, 2012). It is necessary to perform laboratory diagnostic methods (PCR or culture) to accurately detect SD caused by $B$. hyodysenteriae in order to prevent and treat this disease appropriately.

\section{Conclusions}

In this study, 29 positive samples of Brachyspira hyodysenteriae by PCR (11.60\%) in seven provinces of Northern Vietnam were identified. The infection rate differed among regions. The number of SD positive cases was mostly reported in post-weaning pigs in households with poor veterinary hygiene. Pigs 
infected with $B$. hyodysenteriae mainly had the clinical signs of high fever $(100 \%)$ and mucoid diarrhea with grey-brown or bloody feces $(100 \%)$, and gross pathology was concentrated on the large intestine showing swelling and hemorrhagic intestinal mucosa with mucus. PCR diagnosis is essential for accurate disease detection in order to choose effective preventive and treatment measures.

\section{Acknowledgments}

This work was financially supported by research grants from Vietnam National University of Agriculture (VNUA-project No. T2019-03-13VB).

\section{References}

Burrough E. R. (2016). Swine Dysentery: Etiopathogenesis and diagnosis of a re-emerging disease. Veterinary Pathology. 54: 22-31.

Boye M., Baloda S. B., Leser T. D. \& Møller K. (2011). Survival of Brachyspira hyodysenteriae and B. pilosicoli in terrestrial microcosms. Veterinary Microbiology. 81: 33-40.

Carvajal A., Arriba M. L., Rodríguez H., Vidal A. B., Duhamel G. E. \& Rubio P. (2006). Prevalence of Brachyspira species in pigs with diarrhea in Spain. Veterinary Record. 158: 700-701.

Chia S. P. \& Taylor D. J. ( 1978). Factors affecting the survival of Treponema hyodysenteriae in dysenteric pig feces. Veterinary. Record. 103: 68-70.

Vu Dinh Hung \& Doan Khac Huc (1996). Observations of swine dysentery in some industrial rearing farms. Journal of Veterinary science and Technology. 3(2): 56-60. Retrieved from https://www.cabi.org/isc/abstract/19972203231 on November 12, 2020 (in Vietnamese).

Hampson D. J., Fellstrom C. \& Thomson J. R. (2006). Swine dysentery. In: Straw B. E., Zimmerman J. J. \& D'Allaire S. (Eds.). Diseases of Swine. Blackwell Publishing Professional: Ames, IA, USA. 785-805.

Hampson D. J. (2012). Brachyspiral colitis. In: Zimmerman J. J., Karriker L. A., Ramirez A., Schwartz K. J. \& Stevenson G. W. (Eds.). Diseases of Swine (10 $0^{\text {th }}$ ed.). Blackwell Publishing Professional: Ames, IA, USA, 680-696.

Harris D. L. (1984). The epidemiology of swine dysentery as it relates to the eradication of the disease. Compendium on continuing education for veterinarians. 6(2): 83-88.

Jacobson M., Gerth Lofstedt M., Holmgren N., Lundeheim
N. \& Fellstrom C. (2005). The prevalences of Brachyspira spp. and Lawsonia intracellularis in Swedish piglet producing herds and wild board population. Journal Veterinary Medicine. 52: 386-391.

Møller K., Jensen T. K., Jorsal S. E., Leser T. D. \& Carstensen B. (1998). Detection of Lawsonia intracellularis, Serpulina hyodysenteriae, weakly betahemolytic intestinal spirochaetes, Salmonella enterica, and Escherichia coli from swine herds with and without diarrhea among growing pigs. Veterinary Microbiology. 62: 59-72.

Merialdi G., Bonilauri P., Granelli F., Luppi A. \& Dottori M. (2003). Bacterial pathogens in field cases of clinical colitis in growing and finishing pigs in Italy. The Veterinary Record. 153(17): 529-530.

La T., Phillips N. D. \& Hampson D. J. (2003). Development of a duplex PCR assay for detection of Brachyspira hyodysenteriae and Brachyspira pilosicoli in pig feces. Journal Clinical Microbiology. 41(7): 3372-3375.

Ochiai S., Adachi Y. \& Mori K. (1997). Unification of the genera Serpulina and Brachyspira, and proposals of Brachyspira hyodysenteriae Comb. Nov., Brachyspira innocens Comb.Nov. and Brachyspira pilosicoli Comb. Nov. Microbiol Immunology. 41(6 ): 445-452.

Stege H., Jensen T. K., Møller K., Bakbo P. \& Jorsal S. E. (2000). Prevalence of intestinal pathogens in Danish finishing pig herds. Prev. Veterinary Medicine. 46: 279-292.

Taylor D. J. \& Alexander T. J. L. (1971). The production of dysentery in swine by feeding cultures containing a spirochaete. British Veterinary Journal. 127: 58-61.

Thomson J. R., Smith W. J., Murray B. P., Murray D., Dick J. E. \& Sumption K. J. (2001). Porcine enteric spirochete infections in the UK: surveillance data and preliminary investigation of atypical isolates. Animal Research Reviews. 2(1): 31-36.

Yeh J. C., Lo D. Y. \& Chang S. K. (2018). Antimicrobial susceptibility patterns of Brachyspira species isolated in Taiwan. Microbial Drug Resistance. 24: 685-692.

Lugsomya K., Zeeh F., La T., Phillips N. \& Hampson D. J. (2019). First identification and characterisation of Brachyspira hyodysenteriae in pigs in Hong Kong, Porcine Health Management. 5: 27. DOI: 10.11186/s40813-019-0133-x.

Whiting R. A., Doyle L. P. \& Spray R. S. (1921). Swine dysentery. Purdue University Agriculture Experiment Station Bulletin. 257: 3-15.

Walczak K. (2015). Insight into the epidemiology of swine dysentery by analyzing treatment records and using simulation modelling. M.Sc. thesis (Population Medicine), the University of Guelph, Ontario, Canada.

Szancer J. (2019.) Eradication programmes for swine dysentery. International Pig Topics. 20(8): 7-9. Retrieved from http://www.positiveaction.info/pdfs/articles/pt20.8p7. pdf on January 1, 2021. 